\title{
Optical and Infrared Detection Using Microcantilevers
}

\author{
P. I. Oden. E. A. Wachter. P. G. Datskos. T. Thundat and R. J. Warmack \\ Oak Ridge National Laboratory, P.O. Box 2008. Oak Ridge. Tennessee, 37831-6123 \\ Department of Physics. University of Tennessee, Knoxville. TN 37996-1200 (P.I.O., P.G.D., R.J.W.)
}

\begin{abstract}

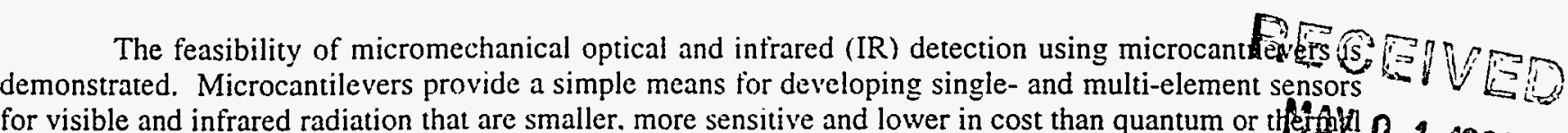
for visible and infrared radiation that are smaller. more sensitive and lower in cost than quantum or theighifl 0,1939 originating from the bimetallic effect. Bending is proportional to the amount of heat absorbed and carbe detected using optical or electrical methods such as resistance changes in piezoresistive cantilevers. 45 microcantilever sensors exhibit two distinct thermal responses: a fast one $\left(\tau_{1}^{\text {thermal }}<m s\right)$ and a slower one $\left(\tau_{2}^{\text {thermal }} \sim 10 \mathrm{~ms}\right)$. A noise equivalent temperature difference. NEDT $=90 \mathrm{mK}$ was measured. When uncoated microcantilevers were irradiated by a low-power diode laser $(\lambda=786 \mathrm{~nm})$ the noise equivalent power, NEP, was found to be $3.5 n \mathrm{~W} / \sqrt{\mathrm{Hz}}$ which corresponds to a specific detectivity, $D^{*}$, of $3.6 \times 10^{7} \mathrm{~cm} \cdot \sqrt{\mathrm{Hz}} / \mathrm{W}$ at a modulation trequency of $20 \mathrm{~Hz}$.
\end{abstract}

\section{INTRODUCTION}

Because infrared is the second most intense radiation band in our environment, its detection and imaging has extensive industrial, military, and commercial applications, including remote monitoring of facilities and equipment, process control, surveillance, night-vision, collision avoidance. and medical imaging. Presently, there are several families of commercially available infrared detectors, including thermopiles, pyroelectrics. bolometers, and various solid state detectors ${ }^{1,2}$. Thermopile detectors typically have a large thermal mass and long response times $(>10 \mathrm{~ms})$. Bolometers using micromachined, suspended foils have much better rise times due to their reduced mass. Both thermopiles and bolometers offer broad spectral response when coated with suitable optically absorbing materials. Solid state detectors for the infrared region, such as quantum well

- devices, must generally be operated at reduced temperatures due to inherently high thermal noise. Additionally, the spectral response of these semiconductor devices is limited by the intrinsic properties of the composing materials. These infrared detectors can be classifies either as quantum - such as the pyroelectrics; or thermal detectors - such as bolometers and thermopiles. For the former type, incident infrared radiation is converted into an electronic response while with thermal detectors, IR radiation is converted into heat which is subsequently detected through temperature changes in the detector. Depending on the operational demands, one type of detection device may be favored over another. As a general rule, when the photon energy of the infrared radiation $h v>k_{B} T$, photon detectors offer better performance and when $h v<k_{B} T$, thermal detectors are generally favored.
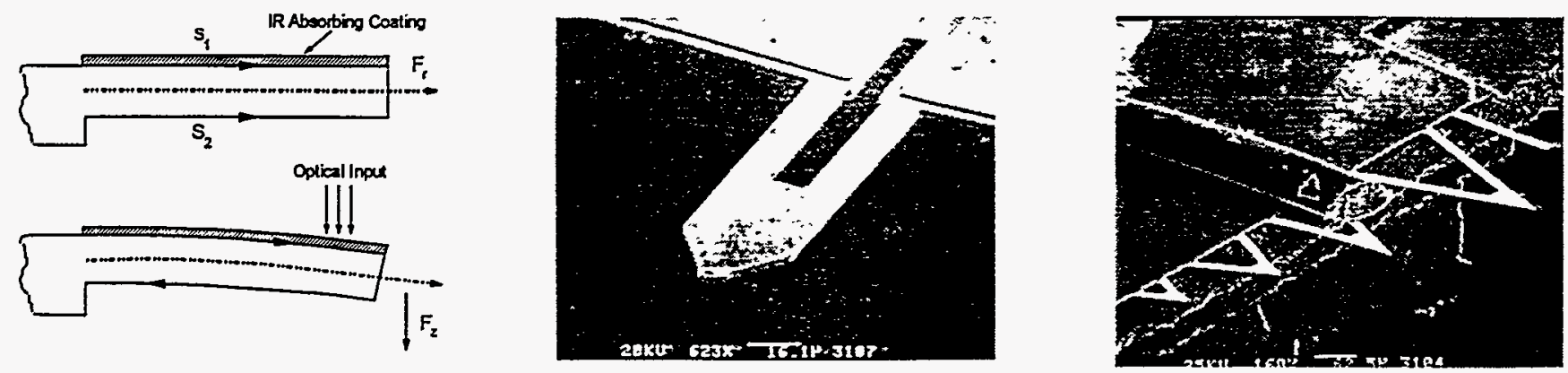

Figure 1. Left drawing is a cross-sectional schematic showing the bending response of a bimetallic cantilever with an IR adsorbing coating. Surface stresses $S_{1}$ and $S_{2}$ are balanced at equilibrium. generatung a radial force $F_{r}$ along the medial plane of the microcantilever. These stresses become unequal upon exposure to IR radiation producing a bending force, $F_{z}$, that displaces the tip of the microcantilever. Middle - is a scanning electron micrograph of one of the piezoelectric IR sensors used (calibration bar is $16 \mu \mathrm{m}$ ). Right - is another scanning electron micrograph of the $\mathrm{Si}_{3} \mathrm{~N}_{4}$ microcantilevers used for the experiments compared to a human hair (calibration bar is $62.5 \mu \mathrm{m}$ ).

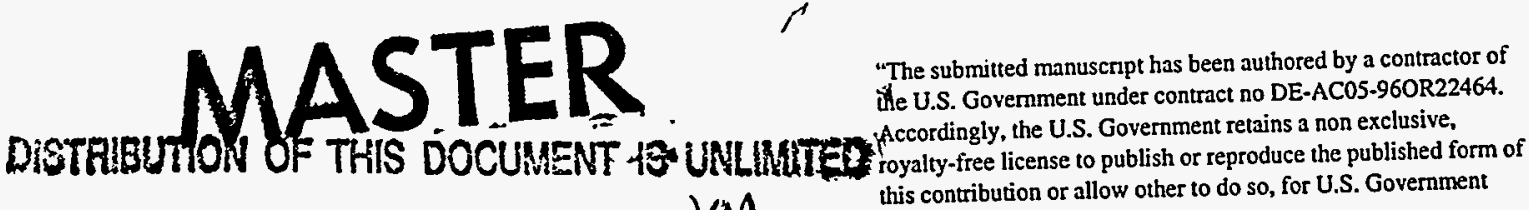

$$
\begin{aligned}
& \text { VM this contribut }
\end{aligned}
$$




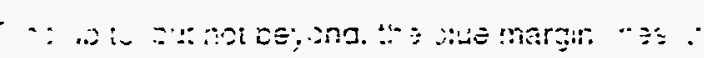

A new approach for producing compact. light-weight, highly-sensitive micromechanical infrared detectors is provided by microcantilever technology which is based on the bending of a microcantilever resulting from absorption of optical energy. When a microcantilever is exposed to infrared radiation, the temperature of the cantilever increases due to absorption of this optical energy ${ }^{3 .+}$. If these microcantilevers are constructed from materials exhibiting dissimilar thermal expansion properties (such as silicon nitride coated with a thin gold film), the bimetallic effect will cause the microcantilever to bend in response to this temperature variation $n^{3-14}$ (such a response is shown in the left image of Figure 1). The extent of bending is directly proportional, in first order. to the rate of energy absorption, which in turn is proportional to the radiation intensity. Previous work has shown that microcantilever bending can be detected with extremely high sensitivity ${ }^{15-17}$. For example, the metalcoated microcantilevers that are commonly employed in atomic force microscopy (AFM) allow sub-Angstrom $\left(<10^{-10} \mathrm{~m}\right)$ sensitivity to be routinely obtained. Recent studies have reported ${ }^{9,14}$ the use of microcantilever bending for calorimetric detection of chemical reactions with energies as low as a few $p J$. It was demonstrated that the detector had an observed sensitivity of $100 \mathrm{pW}$ corresponding to an energy of $150 \mathrm{fJ}$ and proposed using the sensor as a femtojoule calorimeter. An estimate of the minimum detectable power level was of the order of $10 \mathrm{pW}$, corresponding to a detectable energy of $20 \mathrm{fJ}$ and a temperature sensitivity $10^{-5} K^{9}$. However, using an optimally designed cantilever, the sensitivity may be improved even further ${ }^{10-13}$. Hence, for applications in optical radiation detection, microcantilevers can be coated with appropriate absorptive materials such that they undergo bending upon exposure to radiation (such as infrared or near infrared radiation). IR sensing cantilevers are typically $100-200 \mu \mathrm{m}$ long, $0.3-4 \mu \mathrm{m}$ thick and 10-50 $\mu \mathrm{m}$ wide, and made out of materials such as silicon nitride, silicon or other types of semiconducting materials ${ }^{18}$. Due to the monolithic nature of these devices, they can easily be produced in one- and two-dimensional arrays with hundreds of levers on a single wafer. This type of fabrication scheme possesses obvious advantages when considering the production of infrared imaging systems with these cantilever devices.

When considering the bending of the lever, a proportionality between bending and the absorbed energy by the microcantilever is obtained by assuming a spatially uniform incident power, $d Q / d t$, onto a bimetallic microcantilever. Therefore, the maximum deflection, $z_{\max }$, due to differential stress is given by $3,11,13,19$ :

$$
z_{\max }=\frac{5}{4} \frac{\left(t_{1}+t_{2}\right) l^{3}}{\left(\lambda_{1} t_{1}+\lambda_{2} t_{2}\right) w t_{2}^{2}} \cdot \frac{\eta\left(\alpha_{1}-\alpha_{2}\right)(d Q / d t)}{4\left(1+t_{1}^{2} / t_{2}^{2}\right)+1 / t_{1} t_{2}\left(6 t_{1}^{2}+E_{1} t_{2}^{2} / E_{2}\right)+E_{1} t_{1}^{3} / E_{2} t_{2}^{3}}
$$

where $l$ and $w$ are, the length and width of the microcantilever, respectively, $t_{1}$ and $t_{2}$ are the thicknesses of the two layers, $\lambda_{1}, \lambda_{2} ; \alpha_{1}, \alpha_{2} ; E_{1}, E_{2}$ are the thermal conductivities; thermal expansion coefficients and Young's moduli of elasticity of the two layers; $\eta$ is the fraction of the radiation power absorbed.

(a)

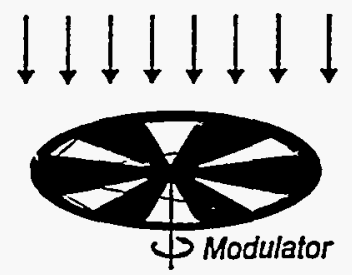

(b)

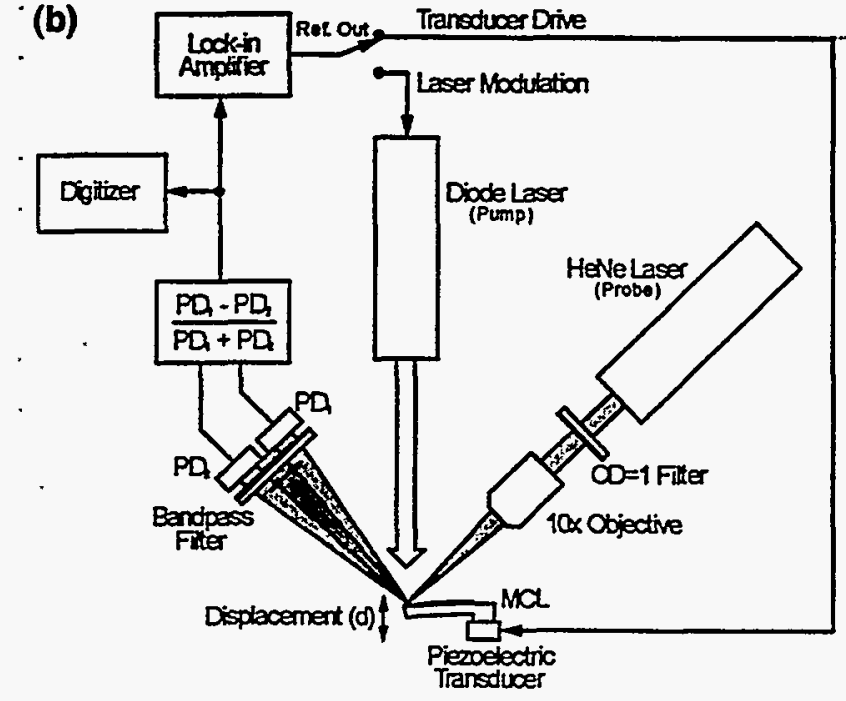

Figure 2. (a) Schematic representation of the piezoresistive IR detection experiment. The piezolever is part of a Wheatstone bridge. one of the legs connected to ground. the other to a metal film resistor with approximately the same resistance $R \sim 2000 \Omega$. The other two outputs are connected to the input of a differential instrumentation amplifier. (b) Schematic diagram of the optical read-out method for IR detection. 

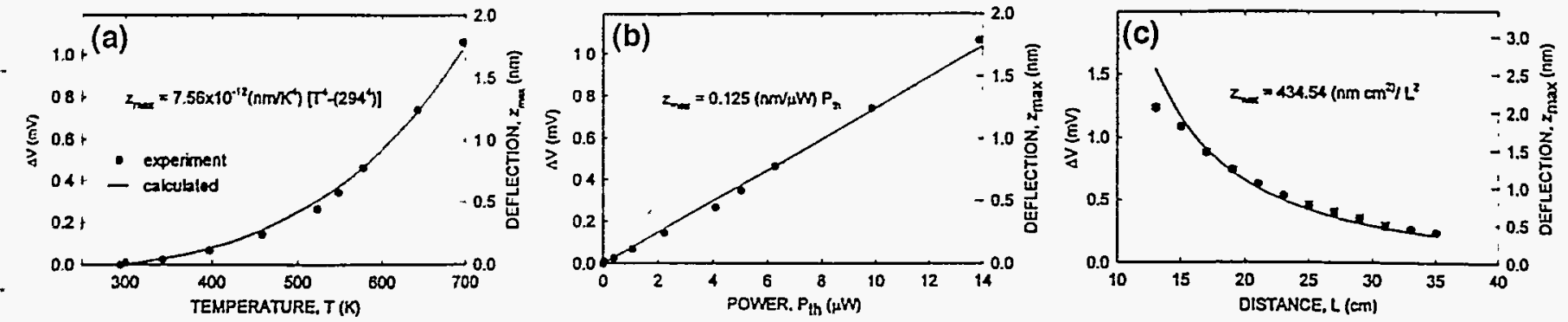

Figure 3. (a) Response of the Wheatstone bndge detection circuit. $J V$. and deflection, $\overline{\max }_{\max }$ of the piezolever as a function of the source temperature. (b) Response as a function of the absorbed thermal power and (c) as a function of IR source distance, $L$.

\section{EXPERIMENTAL}

\subsection{Piezoresistive Deflection Monitoring}

A schematic diagram of the piezoresistive cantilever deflection detection technique is given in Figure 2(a). In this example, surface doped silicon microcantilevers were used in which the piezoresistance across the cantilever varied when it bent due to thermal stimulation. The design and construction of these cantilevers is described in detail elsewhere ${ }^{20.21}$. The total resistance of the cantilever was approximately $2000 \Omega$, which was electrically connected across one arm of a dc-biased Wheatstone bridge circuit. The change in the total resistance is directly proportional to the maximum deflection of the cantilever ${ }^{20}$ :

$$
\Delta R(T)=3 R z_{\max }(T) \times 10^{-6}
$$

- where $z_{\max }(T)$ is expressed in $\mathrm{nm}$.

The thermally induced deflection of the cantilever is caused by the bimetallic effect which arises due to the difference in the thermal properties of the IR coating, the metal layer, and the native silicon body of the cantilever. A reference voltage $V_{0}$ (equal to 9 volts in these experiments) was applied across the circuit and the voltage difference, $\Delta V(T)$, across the Wheatstone bridge circuit was digitized using a Tektronix TDS 544A digital oscilloscope or fed into a Stanford Research Systems SR850 lock-in amplifier. The measured voltage $\Delta V$ is related to the deflection of the cantilever by

$$
\Delta V(T)=\frac{3 V_{0}}{4} z_{\max }(T) \times 10^{6}
$$

The experimental measurements were performed using piezoresistive microcantilevers as temperature sensors in the configuration shown in Figure 2(a). The commercially available piezolever ${ }^{18}$ was coated with $\sim 50 \mathrm{~nm}$ of gold black which served as the IR absorbing material. IR radiation was then focused onto the sensor using a $2.54 \mathrm{~cm}$ diameter IR lens with a focal length of $3.5 \mathrm{~cm}$ and a wavelength transmission range between $0.6-15 \mu \mathrm{m}^{22}$. A Stanford Research Systems SR-540 chopper was used to modulate the IR radiation upon the detector. The sensor assembly was positioned $15 \mathrm{~cm}$ from a soldering iron which served as the IR source. A calibrated thermocouple was attached to the IR source so that its temperature could be recorded.

The thermal power absorbed by the detector can be described as:

$$
P_{\text {thermal }}=\eta \frac{d Q}{d t}=\eta t_{L}\left(\frac{\dot{A_{D}}}{2 \pi L^{2}}\right) A_{S} \varepsilon \sigma_{S-B}\left(T_{S}^{4}-T_{R}^{4}\right)
$$

where $t_{L}$ is the transmission of the lens. $A_{D}$ is the effective area of the sensor, $L$ is the distance of the detector from the source, $A_{S}$ is the area of the target (IR source), $\varepsilon$ is the target's emissivity, $\sigma_{S-B}$ is the Stefan-Boltzmann constant 
$\left(-5.67 \times 10^{-12} \mathrm{~W} \cdot \mathrm{cm}^{-2} \cdot K^{-4}\right), T_{S}$ is the temperature of the target, and $T_{R}$ is the background temperature. In the present studies $t_{L} \sim 0.7, \dot{A_{D}}=6.2 \times 10^{-1} \mathrm{~cm}^{2}, L \sim 15 \mathrm{~cm} . A_{S} \sim 90 \mathrm{~cm}^{2}$, and $T_{R}=294 \mathrm{~K}$. Using these values in Eq. (4), the absorbed thermal power (in Watts) is $P_{\text {thermal }}-6.064 \times 10^{-17}\left[T_{S}^{4}-(294)^{4}\right]$ (assuming that $\eta \sim 0.9$ and a measured $\varepsilon \sim 0.43^{-}$ for the hot iron IR source).

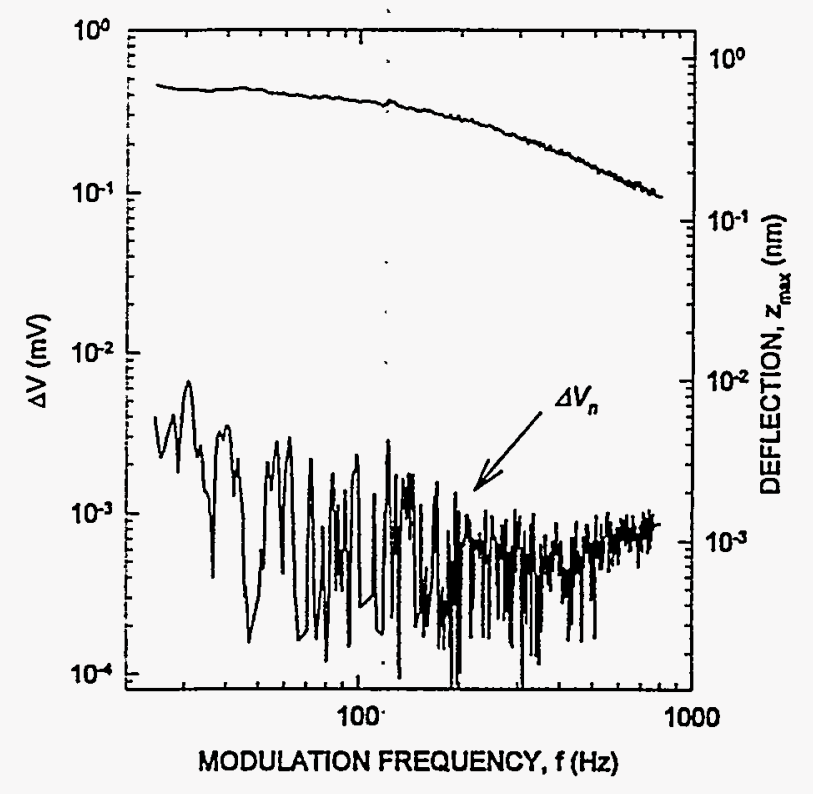

Figure 4. Response of the Wheatstone bridge detection circuit, $\Delta V$, deflection, $z_{\max }$ and noise $\Delta V_{n}$ of the piezolever as a function of the modulation frequency.

The response $\Delta V$ was measured as a function of the temperature, $T$, of the IR source. This is plotted in Figure 3(a) along with the deflection, $z_{\max }$, of the piezolever and can be seen to be a monotonically increasing function of temperature. In Figure 3(b) a plot of the response of Wheatstone bridge circuit, $\Delta V$, and the deflection, $z_{\max }$, of the piezolever detector as a function of the total power absorbed by the detector and it can be seen that it increases linearly with increasing power. From. the slope of this line, a deflection sensitivity of $0.125 \mathrm{~nm} / \mu \mathrm{W}$ is obtained. At a modulation frequency of $30 \mathrm{~Hz}$, a noise equivalent power (NEP) of $\sim 70 \mathrm{nW} / \mathrm{Hz} z^{1 / 2}$ was also obtained, where $N E P=\left(\Delta V_{n} / \Delta V\right) \times P_{\text {thermal }} ; \quad \Delta V_{n}$ is the background noise level $\left(\Delta V_{n} \sim 10^{-6} V\right)$.

The response of the detector was also measured as a function of the distance from an IR source. This is shown in Figure 3(c) where $\Delta V$ and $z_{\max }$ are plotted as a function of the distance, $L$, between the detector and the surface of the soldering iron (IR source); $L$ was varied from $14 \mathrm{~cm}$ to $35 \mathrm{~cm}$. The ambient temperature was $\sim 294 \mathrm{~K}$ and the temperature of the IR source was held at $693 \mathrm{~K}$. The measured $\Delta V$ and calculated $z_{\max }$ were found to decrease with increasing distance and followed closely an inverse square relationship with distance [see Eq. (4)] for distances larger than $15 \mathrm{~cm}$.

Since the response of any thermal sensor depends on both the amount of heat falling onto the detector and the length of time it is exposed to the incoming IR radiation we measured the response of the Wheatstone bridge circuit, $\Delta V$, and the deflection, $z_{\max }$, as a function of modulation frequency of the IR radiation (Figure 4). It can be seen that the detector response (and the deflection of the cantilever) decreases with increasing modulation frequency. The temporal response of the temperature sensor was also determined by measuring $\Delta V$ as a function of time. The microcantilever was found to exhibit two thermal response times due to the incoming IR radiation; a time $\tau_{1}^{\text {thermal }}<1 \mathrm{~ms}$ and a time $\tau_{2}^{\text {thermal }}$ that is somewhat longer $(\sim 10 \mathrm{~ms})$.

These findings demonstrate that smail changes in temperature induce deflections of the microcantilever correspond to measurable changes in the piezocantilever resistance. It should be noted that these commercially available piezolevers have 
been designed to be minimally sensitive to changes in temperature so as to reduce the noise and interference in scanning probe microscopy applications. Therefore, the temperature sensitivity of the piezolever could be further improved by optimizing its shape, IR absorbing coating and thermal isolation.

Type I: $k=0.03 \mathrm{~N} / \mathrm{m}$
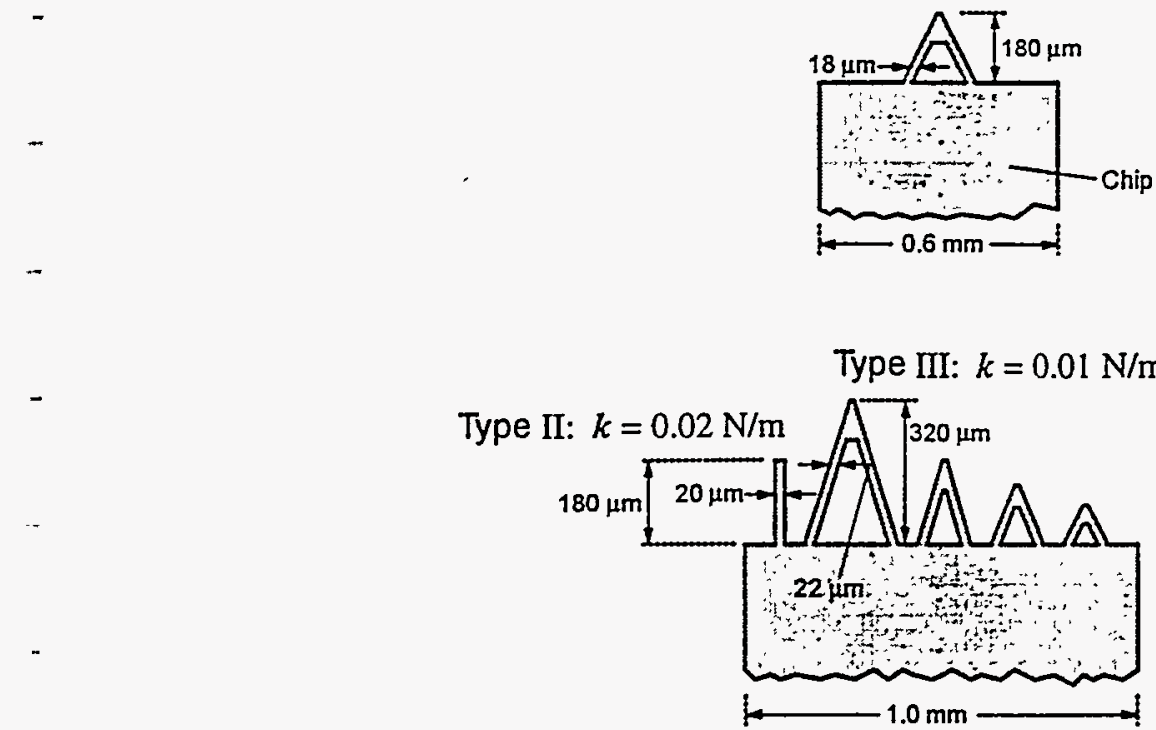

Figure 5. Schematic view of the $0.6 \mu \mathrm{m}$ thick $\mathrm{Si}_{3} \mathrm{~N}_{4}$ microcantilevers used for evaluation of thermal response. Note that results from only the two left-most microcantilevers (out of five total) on the lower chip are presented.

\subsection{Optical Deflection Monitoring}

Bending of microcantilevers can readily be determined by a number of means, including optical, capacitive, tunneling, - and, as shown before, piezoresistive. The optical deflection approach used was adapted from standard atomic force microscopy imaging systems, and is shown in Figure 2(b). Microcantilevers were mounted in a holder (from Digital Instruments) designed for tapping mode AFM, which secured the base of the microcantilever against a small piezoelectric transducer; this chip holder

- was then mounted on a three-axis translation stage to facilitate fine adjustment of the microcantilever relative to the rest of the experimental apparatus. Collimated optical radiation from a diode laser was used to evenly illuminate the mounted microcantilever (pump wavelength of $786 \mathrm{~nm}$, beam diameter of $6 \mathrm{~mm}$, centered on the tip of cantilevers $180-320 \mu \mathrm{m}$ in

- length). Output of this excitation source was modulated sinusoidally at frequencies ranging from $\mathrm{DC}$ to $100 \mathrm{kHz}$, with peak powers ranging from 0 to $18.5 \mathrm{~mW}\left(0\right.$ to $65 \mathrm{~mW} / \mathrm{cm}^{2}$ ). This configuration provided a flexible, easily controlled test system for quantifying microcantilever response to optical energy. All measurements were conducted at ambient temperature and atmospheric conditions.

A second laser was used in a probe configuration to monitor bending. A helium-neon laser (or HeNe, delivering - $3 \mathrm{~mW}$ at $633 \mathrm{~nm}$ ) was focused onto the tip of the microcantilever using a $10 \mathrm{X}$ microscope objective; to minimize heating of the tip by the probe laser, optical power was reduced by placing a neutral density filter with an optical density of 1.0 between the probe laser and the objective. A dual element photodiode displacement detector was used to collect the reflected probe beam

- [position detectors $P D_{1}$ and $P D_{2}$ in Figure 2(b)]; a $1 \mathrm{~nm}$ bandpass filter centered at $633 \mathrm{~nm}$ was placed in front of the detector to block scattered light from the pump laser. The difference signal from the detector pair as the cantilever tip changed position $\left(\left[P D_{1}-P D_{2}\right] /\left[P D_{1}+P D_{2}\right]\right)$ was used to measure the displacement, $d$. This signal was directly digitized and stored, or sent to a lock-in amplifier (SR850, Stanford Research Systems) for signal extraction and averaging. The lock-in amplifier was also used to control modulation frequency and output level of the pump laser. 


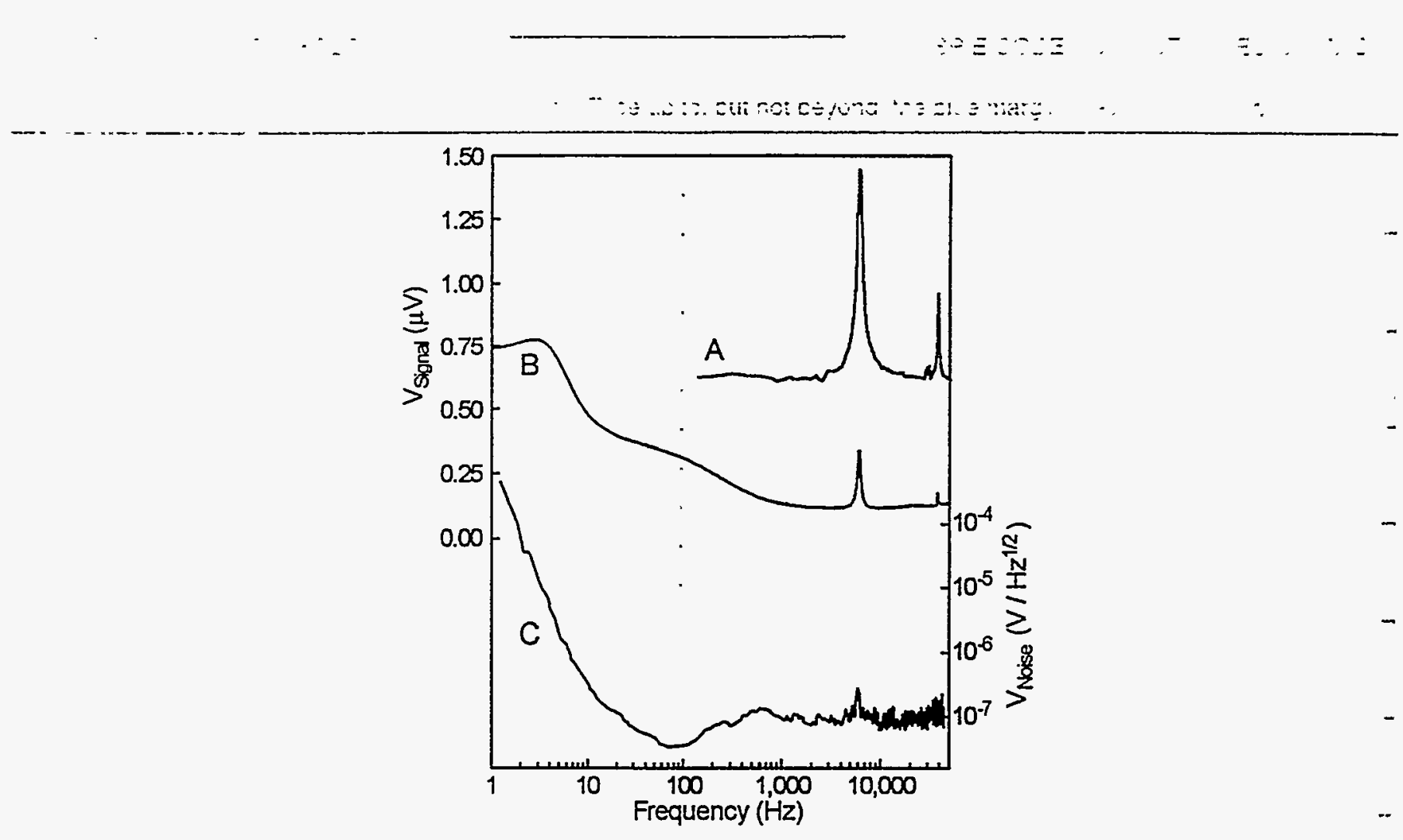

Figure 6. Mechanical and optical excitation spectra for a Type III microcantilever. Response to frequency swept mechanical excitation is given in spectrum 'A': similar results for optical excitation are shown in spectrum 'B'. Optical excitation was effected using a sinusoidally modulated pump laser at $786 \mathrm{~nm}$. Spectrum ' $C$ ' shows noise response when the pump laser is operated in a dc mode. Fundamental mechanical resonance at $6.2 \mathrm{kHz}$ and higher-order resonance at $37 \mathrm{kHz}$ are evident for both optical and mechanical excitation.

Optical response characteristics of three different types of commercially available AFM probe tips were evaluated. These microcantilevers are shown schematically in Figure 5 as well as in the scanning electron micrograph of Figure 1. Microcantilevers typically come from the manufacturer attached to a large rectangular chip (ca. $1 \mathrm{~mm}$ wide $\times 3 \mathrm{~mm}$ long $\times 1 \mathrm{~mm}$ thick) that is used to facilitate manipulation and mounting, and all those evaluated in this work were used as received. The microcantilevers used were: a triangular silicon nitride $\mathrm{Si}_{3} \mathrm{~N}_{4}$ microcantilever (labeled "I" in Figure 5, with a length of $180 \mu \mathrm{m}$, a width of $18 \mu \mathrm{m}$ and a bending force constant $k \sim 0.3 \mathrm{~N} / \mathrm{m}$, from Park Scientific); a rectangular silicon nitride microcantilever (labeled "II", $200 \mu \mathrm{m}$ in length and $20 \mu \mathrm{m}$ in width, bending force constant $k \sim 0.2 \mathrm{~N} / \mathrm{m}$, Park Scientific); and a triangular silicon nitride cantilever (labeled "III", which was $320 \mu \mathrm{m}$ long and $22 \mu \mathrm{m}$ wide, with a bending force constant - $k \sim 0.1 \mathrm{~N} / \mathrm{m}$, Park Scientific). Each was $0.6 \mu \mathrm{m}$ thick. The Type I cantilever was coated with aluminum on one side to see how this would affect its optical response characteristics; Types II and III were used as received from the manufacturer, with a gold/chromium film uniformly covering one side.

An essential aspect of any scheme for micromechanical optical detection is the ability to sensitively detect physical changes resulting from thermal stress, since this directly affects the sensitivity and precision in measurement of temperature change or thermal flux. As an initial evaluation of the ability to detect optically-induced bending of a microcantilever, each of. the three types of microcantilever were subjected to both mechanical and optical excitation, and their response measured as a function of excitation frequency. Mechanical excitation was achieved by driving the piezoelectric element in the AFM chip holder with the reference signal from the lock-in amplifier; such mechanical excitation spectra are helpful in locating resonance frequencies for allowed microcantilever bending modes. Optical excitation spectra were obtained by modulating the pump laser with the lock-in reference signal. Typical response spectra for a triangular microcantilever (Type III) are shown in Figure 6. The mechanical spectrum (curve "A") shows two resonances, at $6 \mathrm{kHz}$ and $38 \mathrm{kHz}$, attributable to the fundamental transverse resonance and a higher-order resonance (possibly torsional bending), respectively. The optical spectrum (curve "B") shows similar resonance features. although with somewhat different relative intensities; a large, broadband response is also noted at low frequencies. No synchronous oscillatory response was noted when the microcantilever was excited with constant dc laser power (curve "C"). Similar response was noted under these conditions for the other two microcantilevers. 


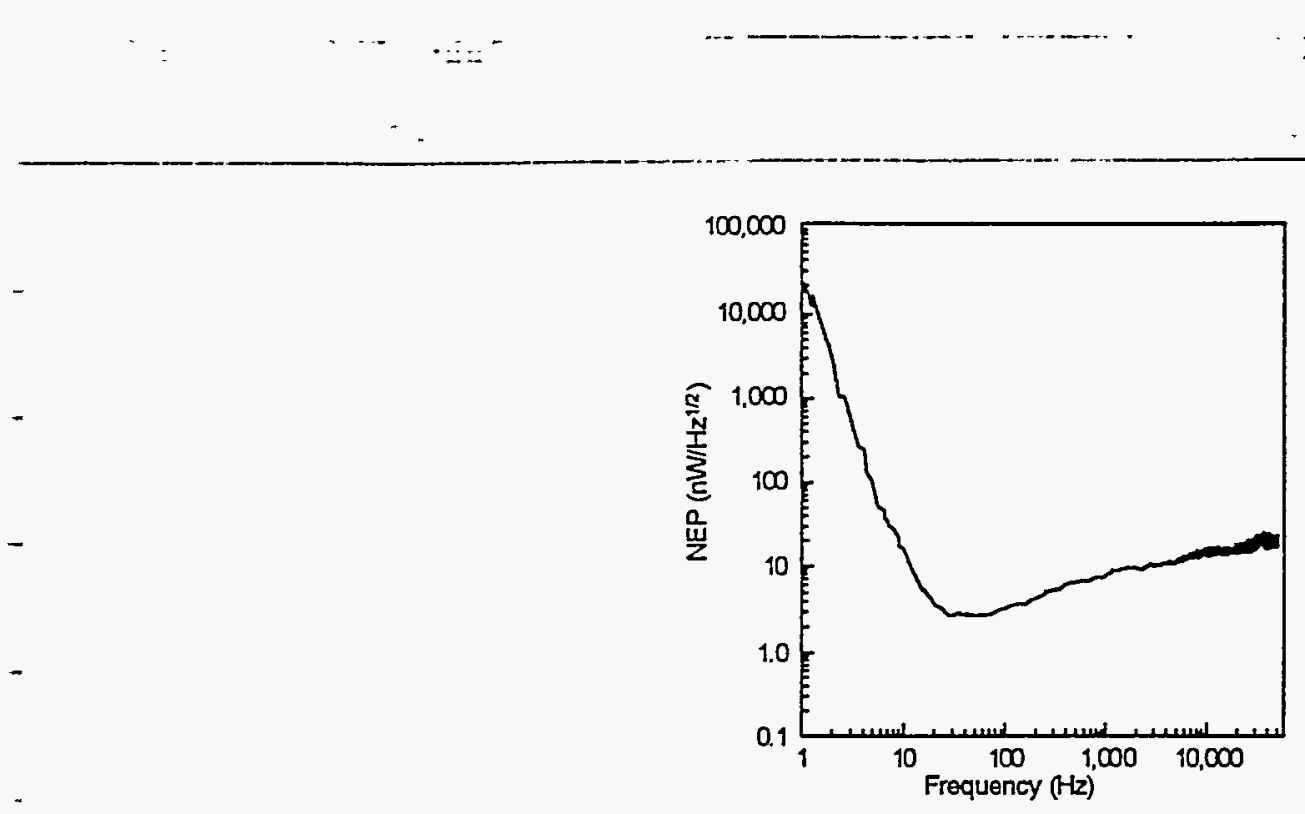

Figure 7. Noise equivalent power (NEP) as a function of modulation frequency for a Type III $\mathrm{Si}_{3} N_{4}$ microcantilever. Optical excitatıon at $785 \mathrm{~nm}$, utilizing bimetallic bending induced in a gold/chromium film.

Figure 6(B) shows that microcantilever response to optical input decreases rapidly for frequencies above $10 \mathrm{~Hz}$, but that mechanical resonance is still observed even at frequencies well above $10 \mathrm{kHz}$. In fact, the Type I and II microcantilevers exhibited strong optical resonance at frequencies of $17 \mathrm{kHz}$ and $14 \mathrm{kHz}$, respectively; these modes correspond to the fundamental transverse resonances for the microcantilevers. Such resonant response demonstrates that reversible heating and bending of the cantilever occurs as a result of optical excitation, producing mechanical vibration. These resonances also have quality factors that are identical to their mechanically-driven counterparts, confirming that optically-pumped mechanical vibration is occurring.

The rapid roll-off in response observed in Figure 6(B) is attributed to thermal equilibration of the cantilever at high modulation frequencies. Because the cantilever must dissipate heat between laser pulses, the finite thermal conductivity of its legs limits the rate at which heat from these thermal impulses can be transferred out of the microcantilever and into the support structure (the chip); thus, as modulation frequency is increased and the microcantilever approaches thermal equilibrium, changes in thermal stress as a function of time approach zero. Comparison of the response of the Type II and Type III cantilevers showed that the rectangular Type II microcantilever had a higher relative response at frequencies above $7.5 \mathrm{kHz}$. We believe this is due to more effective thermal transfer for the rectangular geometry. Since the rectangular microcantilever does not have a. :- geometric restriction between the cantilever and the chip, transfer of thermal energy absorbed at the tip should be more rapid than that for the triangular microcantilever, which has a large area tip suspended on relatively narrow legs. The direct route of thermal transfer in the rectangular microcantilever appears to allow it to maintain a thermal differential at higher optical

- modulation frequencies, and hence to continue responding to the time varying optical stimulus at frequencies well above those practical with the triangular geometry.

- In order to evaluate the role of optical reflectivity (or thermal absorptivity) on microcantilever response, a silicon nitride microcantilever (the Type I specimen) was coated on one side with a thin layer of aluminum; note that the manufacturer's gold/chromium film was removed prior to aluminum deposition. This produced a microcantilever that had a - nearly transparent body that was highly reflective to the pump laser on the aluminum coated side (reflectivity, $R \sim 0.95$ at $786 \mathrm{~nm}$ ), but slightly less reflective on the uncoated side (due to absorption of the pump radiation upon transmission through the $\mathrm{Si}_{3} \mathrm{~N}_{4}$ cantilever body). As expected, the resonant frequency of this cantilever was found to be $17 \mathrm{kHz}$. However, when the - uncoated side of the microcantilever was illuminated (reverse geometry), the magnitude of bending response at all frequencies increased by about $20 \%$ in comparison to normal illumination on the reflective side. We believe this difference is attributable to increased absorption of the pump beam upon transmission through the $\mathrm{Si}_{3} \mathrm{~N}_{4}$ material, resulting in more effective transduction of optical energy into thermal heating of the microcantilever. While this simple experiment demonstrates that sensitivity can be improved by increasing absorption of impinging optical radiation, it is obvious that to optimize the method further suitable optically absorbing coatings are needed (such as carbon black, gold black, or other broadband absorbers). Unfortunately, such materials were not available for this study. 
Photometric response was further characterized by measuring microcantilever response at various modulation frequencies and optical pump levels (Figures 6 and 7. and Table I). For the Type III $\mathrm{Si}_{3} \mathrm{~N}_{4}$ microcantilever, we estimate a

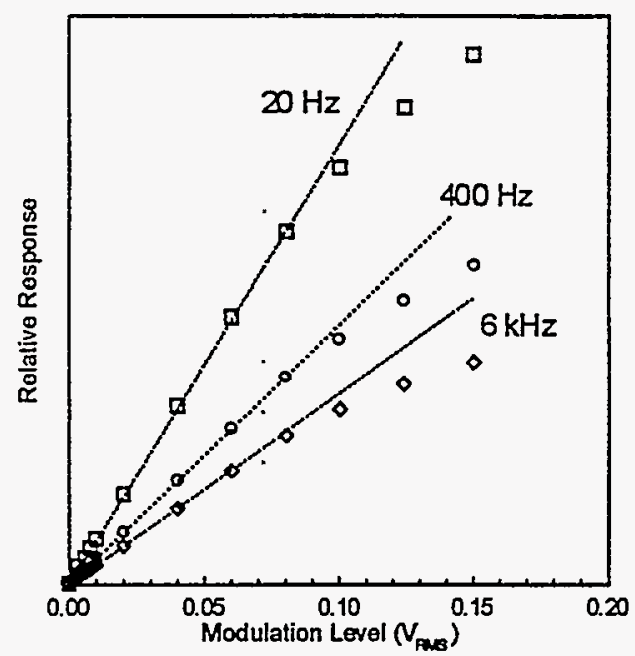

Figure 8. Photometric response for a typical microcantilever (Type III) at various optical pump levels using excitation at $20 \mathrm{~Hz}$, $100 \mathrm{~Hz}$ and $6 \mathrm{kHz}$.

noise equivalent power $(N E P)$ of $3.5 n W / H z$ at $20 \mathrm{~Hz}$, where $N E P=\left(V_{\text {noise }} / B\right)\left(P / V_{\text {signal }}\right), V_{\text {noise }}$ is the background noise level on the cantilever over a lock-in amplifier bandwidth, $B$, of $0.26 \mathrm{~Hz}$, and $P$ is the incident optical power producing an observed signal, $V_{\text {stgnal }}$. Specific detectivity, $D^{*}$, is equal to $3.6 \times 10^{7} \mathrm{~cm} \cdot \mathrm{Hz} z^{1 / 2} / \mathrm{W}$ under these conditions, where $D^{*}=\left(A^{1 / 2} V_{\text {signal }}\right) /\left(V_{\text {noise }} P\right)$, and $A$ is the area of the detector element. Note that the characteristics of this initial, unoptimized microcantilever compare quite favorably with some room temperature technologies currently under development, including indium antimonide photoconductors $(N E P=5 n W \text { at } 500 \mathrm{~Hz})^{23}$, but are not yet competitive with silicon - microbolometers $(N E P=5 p W / H z, \quad N E T D=40 m K \text { at } 30 \mathrm{~Hz})^{24}$ or pyroelectric devices $(N E P=8 p W / \mathrm{Hz}$, $\left.D^{*}=3.5 \times 10^{8} \mathrm{~cm} \cdot \mathrm{Hz}^{1 / 2} / \mathrm{W}\right)^{25}$. However, in contrast to these highly optimized examples, several simple improvements to our microcantilever system are obvious that could improve performance dramatically. For instance, since the metal coating on

Table I. Photometric response at $785 \mathrm{~nm}$ for a gold/chromium coated $\mathrm{Si}_{3} \mathrm{~N}_{4}$ microcantilever (Type III). Data at $6.02 \mathrm{kHz}$ was obtained at the mechanical of the microcantilever.

\begin{tabular}{|c|c|c|c|c|c|}
\hline $\begin{array}{l}\text { Optical Modulation } \\
\text { Frequency }(\mathrm{Hz}) \\
\end{array}$ & $\begin{array}{c}\text { Detector Time Constant } \\
\text { (ms) } \\
\end{array}$ & $\begin{array}{l}V_{\text {topna }} \\
(\mu V)\end{array}$ & $\begin{array}{l}V_{\text {nowe }} \\
(U V) \\
\end{array}$ & $\begin{array}{c}\text { NEP } \\
(\mathrm{n} w / \sqrt{ } / \mathrm{H} z) \\
\end{array}$ & $\begin{array}{c}\mathrm{D}^{*} \\
\left(\mathrm{~cm} \cdot \mathrm{Hz} \mathrm{z}^{1 / 2} \mathrm{M}\right) \\
\end{array}$ \\
\hline \multirow[t]{3}{*}{6020} & 300 & 307.1 & 0.585 & 13.1 & $9.48 \times 10^{8}$ \\
\hline & 30 & 305.3 & 1.76 & 12.5 & $9.91 \times 10^{8}$ \\
\hline & 1 & 305.5 & 9.46 & 12.3 & $1.01 \times 10^{7}$ \\
\hline \multirow[t]{3}{*}{400} & 300 & 223.2 & 0.196 & 6.03 & $2.06 \times 10^{7}$ \\
\hline & 30 & 221.1 & 0.691 & 6.78 & $1.83 \times 10^{7}$ \\
\hline & 10 & 226.3 & 1.19 & 6.59 & $1.88 \times 10^{7}$ \\
\hline 20 & 300 & 528.2 & 0.266 & 3.46 & $3.59 \times 10^{7}$ \\
\hline
\end{tabular}

the tested cantilevers is highly reflective at the pump wavelength (for gold, $R>98 \%$ at $785 \mathrm{~nm}$ ), use of an improved absorptive coating (such as gold black. $R<2 \%$ ) could improve NEP in this example to $<75 p \mathrm{~W}$. Furthermore, the observed 
detection limits appear to be determined by readout noise in our optical detection circuit. We believe that with careful design of this circuitry, performance could be substantially improved. Finally, response of the microcantilevers was extremely linear (with a correlation coefficients, $r_{2}>0.99995$ ) for all but the highest test levels; roll-off in measured response for very high

- laser modulation levels is an artifact of our method for modulating the pump laser, which exhibited a reduced depth of modulation at high drive levels.

\section{CONCLUSIONS}

We have demonstrated that microcantilevers represent an important development in room temperature infrared detector

- technology, and can be expected to provide the basis for considerable further development. For example, while the microcantilevers employed here were optimized for standard AFM applications (and were in fact designed to minimize thermal sensitivity), vastly improved detectors could be produced by making relatively simple changes in the materials and geometries

- used in microcantilever fabrication. It is possible to design microcantilevers with much smaller force constants by varying the geometry of the cantilever, and in contrast to the devices used in this study, cantilevers with force constants as small as $0.006 \mathrm{~N} / \mathrm{m}$ are now commercially available. Since the fundamental mechanical resonance frequency of a microcantilever is

- proportional to $\sqrt{k}$, reductions in force constant can be used to bring resonance into ranges compatible with mechanical chopping frequencies. It is also clear that the coatings applied to the cantilever are at least as important as the composition of the cantilever itself. For example, high thermal expansion bimetallic coatings (such as films of $\mathrm{Al}, \mathrm{Zn} . \mathrm{Pb}$, or In) could be

- used to increase the thermally-induced bending of the cantilever. Coating the surface of the cantilever with high emissivity materials (such as gold black) can also enhance IR response.

Since microcantilever spectral response can be easily tailored through the application of specific absorptive coatings, choice of material for fabrication of the microcantilever can be determined primarily by the requirements of the manufacturing process. This means that microcantilevers can be fabricated using standard semiconductor methods and materials, and as a consequence could be mass produced at very low cost. Hence, two-dimensional cantilever arrays based on the technology

- described here could become very competitive with existing technologies due to their inherent simplicity, high sensitivity, and rapid response to optical radiation. While the optical readout method is useful with single element designs, practical implementation of microcantilever arrays may require the use of other readout methods, such as piezoresistance. Fortunately,

- the microcantilever technology's compatibility with a variety of readout methods also affords tremendous flexibility to potential system designers.

\section{ACKNOWLEDGEMENTS}

Research sponsored by U.S. Department of Energy under contract DE-AC05-96OR22464 with Lockheed Martin Energy Research Corp. The authors wish to acknowledge the support of the ORNL Seed Money Program. The efforts of P.I. Oden were supported, in part, by an appointment to the Alexander Hollaender Distinguished Postdoctoral Fellowship Program sponsored by the U.S. Department of Energy, Office of Health and Environmental Research, and administered by the Oak Ridge Institute for Science and Education. P.G. Datskos wishes to acknowledge the support of the National Science Foundation under the SBIR award DM2-9460929 to Consultec Scientific, Inc.

$-$

\section{REFERENCES}

1. R .J. Kayes, Editor "Optical and Infrared Detectors," Topics in Applied Physics, Volume 19, Springer Verlag Berlin (1977).

2. P. Howard, J. Stevens, C. Rau, R.J. Herrring, and R.A. Wood, Infrared Technology XXI, SPIE Vol. 2552-60, July 1995.

3. J.R. Barnes, R.J. Stephenson, C.N. Woodburn, and M.E. Welland, Rev. Sci. Instum. , 65, 3793 (1994).

- 4. O. Nakabeppu, M. Chandrachood, Y. Wu, J. Lia, and A. Majumdar, Appl. Phys. Lett. , 66, 694 (1995).

5. N. Umeda, S. Ishizaki, and H. Uwai, J. Vac. Sci. Technol. , B9, 1318 (1991).

6. M. Allegrini, C. Astoli, P. Baschieri, F. Dinneli, C. Frediani, A. Lio, and T. Mariani, Ultramicroscopy, 42-44, 371 (1992).

7. O. Marti, A. Ruf, M. Hipp, B. Bielefeldt, J. Colchero. and J. Mlynek, Ultramicroscopy, 42-44, 345 (1992).

8. J. Mertz, O. Marti, and J. Mylnek, Appl. Phys. Lett. 62. 2344 (1993).

9. J. K. Gimzewski, Ch. Gerber, E. Meyer, and R. R. Schlitter. Chem. Phys. Lett., 217, 589-594 (1994).

10. T. Thundat, R. J. Warmack, G. Y. Chen, and D. P. Allison. Appl. Phys. Lett., 64, 2894-2896 (1994). 


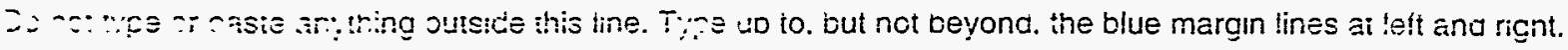

11. E. A. Wachter, T. Thundat, P. G. Datskos, P. I. Oden, S. L. Sharp, and R. J. Warmack, "Remote Infrared Detection Using Microcantilevers", Rev. Sci. Instrum. (submitted).

12. G. Y. Chen, T. Thundat, E. A. Wachter, R. J. Warmack. "Ảdsorption-Induced Surface Stress and Its Effect on Resonance Frequency of Microcantilevers," J. Appl. Phys., 77, 1 (1995).

13. P.G. Datskos, P.I. Oden, T. Thundat, E.A. Wachter, R.J. Warmack and S.R. Hunter, "A Micromechanical Temperature Sensor", Appl. Phys. Lett. (submitted).

- 14. J. K. Gimzewski. Ch. Gerber, E. Meyer, and R. R. Schlitter, "Micromechanical Heat Sensor: Observation of a Chemical Reaction, Photon and Electrical Heat Pulses", in Forces in Scanning Probe Methods, Proceeding of the Nato ASI, H.-J. Güntherodt, D. Anselmetti, and E. Mayer (eds.) p. 123-131 (1995).

- 15. D. Sarid. Scanning Force Microscopy, Oxford University Press, New York, (1991).

16. "Investigations of the Reconstructed Gold Surface with Electrochemical Scanning Probe Microscopy" - PhD. dissertation, Patrick Ian Oden, Arizona State Univesity Physics Department (1993).

17. P.I. Oden, N.J. Tao and S.M. Lindsay, J. Vac. Sci. Technol. B11(2), 137 (1993).

18. Commercial versions available through Digital Instruments, Santa Barbara, CA and Park Scientific, Sunnyvale, CA.

19. W. C. Young, Roark's Formulas for Stress and Strain. Sixth ed., McGraw Hill, New York, pp. 118 (1989).

20. M. Tortonese, R. C. Barrett, and C. F. Quate, Appl. Phyis. Lett., 62, 834 (1993).

- 21. F. J. Giessibl and B. M. Trafas, Rev. Sci. Instrum., 65, I923 (1994).

22. Oriel Co., Model 43402 AMTIR-1 lens.

23. R. Anton, E.L Dereniak, and J. Garcia. Infrared Technology XXI, SPIE Vol. 2552, 592, July 1995.

- 24. N. Butler. R. Blackwell, R. Murphy, R. Silva, and C. Marshall, Infrared Technology XXI, SPIE Vol. 2552, 583, July 1995.

25. S. Fujii. T. Kamada, S. Hayashi, Y. Tomita, R. Takayama, T. Hirao, T. Nakayama, and T. Deguchi, Infrared Technology

- $\quad$ XXI, SPIE Vol. 2552, 612, July 1995. 


\section{DISCLAIMER}

This report was prepared as an account of work sponsored by an agency of the United States Government. Neither the United States Government nor any agency thereof, nor any of their employees, makes any warranty, express or implied, or assumes any legal liability or responsibility for the accuracy, completeness, or usefulness of any information, apparatus, product, or process disclosed, or represents that its use would not infringe privately owned rights. Reference herein to any specific commercial product, process, or service by trade name, trademark, manufacturer, or otherwise does not necessarily constitute or imply its endorsement, recommendation, or favoring by the United States Government or any agency thereof. The views and opinions of authors expressed herein do not necessarily state or reflect those of the United States Government or any agency thereof. 


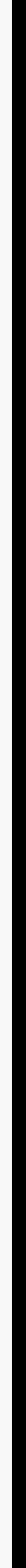

